\title{
Virtual Reality in the Management of Chronic Low Back Pain: A Scoping Review
}

\author{
Ameet S. Nagpal ${ }^{1 \star 1}$, Aditya Raghunandan ${ }^{2}$, Faye Tata $^{2}$, Daniel Kibler ${ }^{2}$ and \\ Donald McGeary ${ }^{3}$
}

${ }^{1}$ Department of Anesthesiology, UT Health San Antonio, San Antonio, TX, United States, ${ }^{2}$ Department of Rehabilitation Medicine, UT Health San Antonio, San Antonio, TX, United States, ${ }^{3}$ Department of Psychiatry, UT Health San Antonio, San Antonio, TX, United States

OPEN ACCESS

Edited by:

Cletus Cheyuo,

University of Toronto, Canada

Reviewed by:

Brandon Birckhead,

Johns Hopkins Medicine,

United States

*Correspondence:

Ameet S. Nagpal

ameet.nagpa/@gmail.com

†Present address:

Ameet S. Nagpal,

Department of Orthopaedics and

Physical Medicine, Medical University

of South Carolina, Charleston, SC,

United States

Specialty section:

This article was submitted to Neuromodulatory Interventions,

a section of the journal

Frontiers in Pain Research

Received: 17 January 2022 Accepted: 31 January 2022

Published: 07 March 2022

Citation:

Nagpal AS, Raghunandan A, Tata F, Kibler D and McGeary D (2022) Virtual Reality in the Management of Chronic

Low Back Pain: A Scoping Review. Front. Pain Res. 3:856935.

doi: 10.3389/fpain.2022.856935
Virtual reality (VR) is a burgeoning treatment option for chronic pain. Its use has been heterogenous in the literature. This scoping review assesses the current literature for the use of VR in the treatment of chronic low back pain (CLBP). The following themes were identified by the analysis: safety and feasibility of VR, quality of life associated with VR treatment for CLBP, efficacy of VR to treat CLBP, and efficacy of VR to treat functional changes associated with CLBP. Gaps were identified after analysis of the extant literature. Although the nascent research uncovered in this scoping review found good evidence for safety and tolerability of VR, more studies of safety, acceptance, and satisfaction are recommended including focused studies of spinal pain risks specific to use of VR. Overall, the methodological quality of studies reviewed in this scoping review was poor and outcomes were limited to short-term posttreatment outcomes.

Keywords: chronic low back pain, virtual reality, augmented reality, low back pain, scoping review

\section{INTRODUCTION}

Non-specific low back pain (NSLBP) is defined as low back pain not attributable to a known cause, whereas chronic low back pain (CLBP) refers to a myriad of potential etiologies which cause ongoing pain for longer than 12 weeks (1-3). In the US, the prevalence of chronic low back pain in the adult population (over the age of 18) ranges between 10 and 30\% with a lifetime prevalence as high as $65-80 \%$ (4). These high prevalence rates correspond to an associated significant socioeconomic burden in the United States (US). A retrospective study performed by Mehra et al. analyzed nationwide integrated medical/pharmaceutical claims data and revealed that the total direct costs of CLBP-related resource use were $\sim \$ 96$ million over a 12-month follow-up for all CLBP patients, with a mean annual cost of $\$ 2,426$ per patient (5).

Given this high burden of care, it is prudent to provide the most cost-effective and evidencebased diagnosis and treatment options to patients with NSLBP and CLBP. The recent North American Spine Society (NASS) 2020 Clinical Guideline for the Diagnosis and Treatment of Low Back Pain addresses a variety of evidence-based recommendations for the management of non-specific low back pain. Providing an accurate diagnosis for the etiology of CLBP can clarify management recommendations, however, this may not be possible in patients where non-structural causes of low back pain may be considered. A recommended multimodal non-interventional approach to management of CLBP includes: cognitive behavioral therapy, patient education, treatments targeting fear avoidance, McKenzie exercise [directional preference, centralization and mechanical diagnosis and therapy (MDT)], yoga, aerobic exercise, work hardening or conditioning, non-selective NSAIDs, judicious use of opioid medications. Interventional management options 
include radiofrequency neurotomy of medial branches of the lumbar spine for facet-mediated pain, intra-articular joint injections or lateral branch neurotomy for sacroiliac joint pain, and several experimental procedures for discogenic pain (6). For patients who do not improve with these measures, surgical management may be an option.

In addition to the non-surgical options detailed above, extended reality (XR) technologies provide a new alternative to medication and interventions for management of CLBP. $X R$ is an umbrella term that describes emerging immersive technologies that can merge the physical and virtual environments. Some of the common immersive technologies used for pain management include virtual reality (VR), augmented reality (AR), and mixed reality (MR) (7). VR was adopted early by the gaming and entertainment industry to provide a fully immersive experience into a simulated digital environment. Individuals must wear a VR head-mounted display to obtain an immersive feel for an artificial world. VR has been shown as an effective treatment option for managing acute pain, but the body of research for chronic pain is still in the early stages (8). In AR, however, virtual information and/or objects can be overlaid on the real world. This virtual information can enhance the physical world with digital details such as images, text, and animation. These virtual details can be delivered via glasses, screens, tablets, and smartphones. AR has been used as a safe way to help trainees practice routine and complex spine surgical techniques (9). Finally, in MR virtual and physical objects co-exist and can interact with one another in real-time. This is the latest immersive technology and alternatively referred to as hybrid reality for which a specific MR headset is required. The scope for this technology is vast with applications in medical education training, research, communication, and clinical treatment (10).

Virtual reality offers novel immersion to pain rehabilitation that may enhance outcomes from other rehabilitation protocols (as an adjunctive technology) and provide direct analgesic effects through yet unspecified mechanisms (11). However, the body of research examining VR for CLBP is nascent and early systematic reviews found little evidence supporting incremental benefit of physical rehabilitation with adjunctive VR (12). Research on VR for pain rehabilitation is rapidly growing, so this scoping review will specifically address the latest available evidence for VR as it relates to management of CLBP, identify and analyze current knowledge gaps, and serve as precursor to future systematic reviews.

\footnotetext{
Abbreviations: 6MWT, 6-Minute Walk Test; ACTH, Adrenocorticotropic hormone; ASQOL, Ankylosing Spondylitis Quality of Life Questionnaire; BASFI, The Bath Ankylosing Spondylitis Functional Index; BDI-II, Beck Depression Inventory-II; CPAQ-8, Chronic pain Acceptance Questionnaire; DVPRS, Defense and Veterans Pain Rating Scale; HOMA-IR, homeostatic model assessment insulin resistance; IKT, Isokinetic training; MPQ, McGill Pain Questionnaire; NPRS, numeric pain rating scale; ODI, Oswestry Disability Index; PCS, patient catastrophizing scale; PGIC, patient's global impression of change; PSEQ2, Pain Self-Efficiency Questionnaire; ROM, range of motion; RMDQ, Roland Morris Disability Questionnaire; SF36, (RAND corporation) Short form 36 item health survey; SUS, System Usability Scale; TEI, Treatment Evaluation Inventory; TUG, Timed-up and go test; VRT, virtual reality training.
}

\section{METHODS}

\section{Design}

To understand the available range of extant research on the topic of VR as a treatment for CLBP, this scoping review followed the required 5 steps of a previously published framework (13). The five steps followed included:

1. Identification of the research question ("what does existing data demonstrate on the topic of utilizing VR for the treatment of CLBP”)

2. Identification of relevant studies

3. Study selection

4. Charting of data

5. Collating and summarizing and reporting findings

After following this framework, our team analyzed available literature in an attempt to develop themes, describe the current state of the literature, and identify research gaps.

\section{Search Strategy}

Medline and Scopus were searched by members of the research team (ASN, AR, FT, and DK) utilizing the search string of ("virtual reality" AND "chronic low back pain") with filters applied for human studies (no search suffixes used). These searches were performed at various intervals in July, August, September, and October of 2021. Forty-eight articles were initially identified by this strategy. Thirty articles were then excluded (11 were duplicates, 24 were excluded because they did not meet inclusion criteria), and so 13 total studies were included (14-26).

\section{Inclusion Criteria}

Studies were included if VR was utilized for a study population with chronic pain primarily of the low back. English and non-English language studies were acceptable. Randomized controlled trials, prospective and retrospective cohort studies, and observational studies were included. Case reports and ongoing studies were excluded.

\section{Development of Themes}

After the final included manuscripts were identified, each team member was responsible for reading each of these manuscripts. The team then met asynchronously on 3 different occasions to identify grouped themes associated with the published literature on the topic of VR and CLBP. A final asynchronous meeting occurred in order to identify literature gaps. There were no disagreements and therefore moderation for resolution was not necessary.

\section{RESULTS}

The following themes were identified by the analysis: safety and feasibility of VR, quality of life associated with VR treatment for CLBP, efficacy of VR to treat CLBP, and efficacy of VR to treat functional changes associated with CLBP. A summary analysis of all included articles to assess if the articles included a clinical trial, randomized, included a control, and justification for sample 
size was completed (Table 1). Although all included articles were clinical trials, two were single armed studies, one study acting as a proof-of-concept and the other to further validate its specific VR program. Most (eight out of thirteen) articles justified their chosen sample size ranging from 13 participants (14), to 179 participants (21). On average, sample size was approximately 50 .

\section{Safety and Feasibility of VR}

Four different studies employed unique scales and markers to observe the fundamental feasibility and safety of VR.

Hennessey et al. used the System Usability Scale (SUS), a 10item survey specifically focusing on usability of a VR app, and the Treatment Evaluation Inventory (TEI), a 9-item questionnaire regarding acceptability of this VR program. Scores greater than or equal to the cut-off TEI score were observed in $92 \%$ of participants and $75 \%$ of participants scored above average for SUS, demonstrating the usability, or feasibility of this app (14).

In Thomas et al.'s study, participants rated their overall impressions of the VR game using a survey that was adapted from an existing measure of online health game acceptability, the Game Experience Survey. The Roland-Morris Disability Questionnaire (RMDQ) and McGill Pain Questionnaire (MPQ) were utilized, along with participant retention rate and use of pain medications, to examine safety and feasibility of their dodgeball VR program. The Game Experience Survey ratings suggested a high level of acceptability for the game with strong ratings of enjoyment combined with willingness to continue to play the game and recommend it to others with back pain. The RMPQ and MPQ demonstrated that regardless of study arm, participants did not have a change in physical disability over time and pain intensity did not increase. Only one participant took pain medication for back pain preemptively prior to a VR session and $100 \%$ retention of participants was observed (15).

Nambi et al. studied VR vs. isokinetic training in football players with CLBP, all groups showed significant improvements in all observed stress hormone levels, with exception of glucose and insulin, after 4 weeks of intervention. Insulin resistance, growth hormone, prolactin, АCTH, and cortisol showed significant improvements all around at 6 months follow up, although greater tendency toward improvement in the VR group was noted per the post hoc Bonferroni test (16). Likewise, when Nambi's team studied soccer players with CLBP, there were significant improvements in all stress hormones without HOMAIR in all studied groups at 4 weeks. Significant improvements in glucose, prolactin, ACTH, and cortisol were observed in all groups at 6 months, although more so in the VR group (26).

\section{Quality of Life Associated With VR Treatment for CLBP}

Four articles evaluated the impact of virtual reality on the participants' quality of life (QOL).

Nambi et al. used a player wellness questionnaire at baseline, at 4 weeks, 8 weeks, and 6 months of VR that utilizes a five-point Likert scale to assess fatigue, sleep quality, muscle soreness, stress, and mood. A post hoc analysis, and percentage of improvement between groups were statistically significant for the VR group showing improvement in wellness/quality of life (17).
Park assessed quality of life using the short form health survey (SF36). The SF36 is a 36-item participant reported survey, and within the 36 items there are two categories, physical health, and mental health. Aspects in the physical health category are physical functioning, role limits from physical health, pain, and general health perception. Mental health categories address emotional well-being, role limits due to mental health, social functioning, and energy level/fatigue. The Nintendo Wii (VR) group improved significantly in terms of role limitations due to mental health, energy/fatigue, and emotional well-being (18).

Karahan et al. measured QOL with the ankylosing spondylitis quality of life questionnaire. This is an eighteen-item disease specific questionnaire designed to address disease impact on aspects of life such as sleep, mood, motivation, coping, daily activities, independence, relationships, and social life. After the eight-week intervention the ASQOL scores improved significantly in the virtual reality group (19).

Alemanno et al. used the SF36 and the Beck Depression Inventory (BDI-II) to assess QOL. The The single arm treated with VR showed significant improvements in five of the eight subscale scores within the SF36: physical functioning, physical role functioning, bodily pain, vitality, and social role functioning. A significant improvement was seen in post treatment BDI-II scores (20).

\section{Efficacy of VR to Treat Chronic Low Back Pain (CLBP)}

Ten studies studied the efficacy of virtual reality to treat CLBP. See Table 2 for further details.

At three points during Garcia et al.ss study, pre-intervention, during, and post intervention, average pain intensity was measured using the Defense and Veterans Pain Rating Scale (DVPRS). DVPRS uses a ten-point Likert scale to address how pain affects activity, sleep, mood, and stress. Pain improvement was measured with the Patient Global Impression of Change Scale (PGIC). By the end of the study, the VR group had significantly lower average DVPRS scores than the sham VR group and significant improvement in PGIC compared to the sham VR group. No significant differences between groups were observed for pain catastrophizing, pain self-efficacy, and pain acceptance (21).

Matheve et al. measured pain using the numeric pain rating scale (NPRS) and PCS. This study noted significant reduction in pain intensity during and after exercise for the VR group compared to the control. No significant changes were seen with pain catastrophizing and baseline pain intensity (22).

Multiple studies, including Yilmaz, Kim, Yoo, Karahan, Park and the three articles by Nambi used the visual analog scale (VAS) to measure VR efficacy to treat CLBP. Each study showed a significant improvement in VAS pain scores for the VRT group compared to the control group (16-19, 23-26). In Park et al.'s study, lumbar stabilizing exercise group and VR group both showed a significant reduction in VAS pain scores when compared to the control group (18). In Nambi's article that compared Virtual reality vs. isokinetic training to assess its effect on pain, kinesiophobia, and serum hormones, the 
TABLE 1 | Summary analysis of all included articles to assess for study rigor.

\begin{tabular}{|c|c|c|c|c|c|}
\hline 1st Author & Reference \# & Clinical trial? & Randomized? & Controlled? & Sample size justification? \\
\hline Hennessy & (14) & Yes & No & No & No \\
\hline Thomas & (15) & Yes & Yes & Yes & Yes \\
\hline Nambi & (16) & Yes & Yes & Yes & Yes \\
\hline Nambi & (17) & Yes & Yes & Yes & Yes \\
\hline Park & (18) & Yes & Yes & Yes & No \\
\hline Karahan & (19) & Yes & Yes & Yes & No \\
\hline Alemanno & (20) & Yes & No & No & Yes \\
\hline Garcia & (21) & Yes & Yes & Yes & Yes \\
\hline Matheve & (22) & Yes & Yes & Yes & Yes \\
\hline Yilmaz & (23) & Yes & Yes & Yes & Yes \\
\hline Kim & (24) & Yes & Yes & Yes & No \\
\hline Yoo & (25) & Yes & Yes & Yes & No \\
\hline Nambi & (26) & Yes & Yes & Yes & Yes \\
\hline
\end{tabular}

post hoc Bonferroni test and graphical representation showed improvement in pain intensity in the VRT and IKT group in comparison to the control group (16).

\section{Efficacy of VR to Treat Changes in Function Associated With CLBP}

Eight studies assessed the ability of VR to treat various components of functional changes associated with CLBP. See Table 2 for further details.

Outside of VR gameplay, Thomas et al. observed no significant effects with group (game vs. control) on change in lumbar flexion from initial standing posture to target contact (extracted with custom software) (15).

Alemanno et al. looked at kinematic data using a tracking system and measured the maximal and average truncal range of motion and an index of proprioception ranging from 0 to 1 , where 1 corresponded to the maximal accuracy in trunk rotation. The statistical analyses of RMDQ and these kinematics data revealed a significant improvement in participation, trunk functionality, average range of motion, and proprioception after VR intervention (20).

Yoo et al. used an isokinetic dynamometer for measurements of force generated by the trunk. During trunk extension and flexion, peak torque (or rotational force) and total work were measured at specific angular speeds (30 degrees per second and 90 degrees per second, respectively). This data was used along with body weight, fat mass, and muscle mass to compute additional data to relate force measurements to a subject's body composition. After 8 weeks, peak torque and total work were significantly enhanced only in the horse simulator riding group and decreased in control group. Mentioned related ratios correlated (25).

In Yilmaz et al.'s study, participants of each group were evaluated for disability with the Oswestry Disability Index (ODI), and functional capacity with the Timed Up (TUG) and Go test plus 6-Minute Walk Test (6MWT). Balance ability was assessed with the Single-leg balance test. After treatment, there was a significant difference in the TUG and 6MWT scores between control and VR groups. There was no significant difference in ODI or balance tests between groups, but there was significant difference before and after treatments in each group (23).

Likewise, Kim et al. utilized ODI and RMDQ to examine functional level during pain and severity of disability with significant improvements between groups in pre and post training ODI scores. This study found significant differences in ODI and RMDQ scores between groups regarding effect of time-by-group interaction (24).

Before and after 8 weeks of intervention, Park et al.'s participants were evaluated for back strength and balance ability using the maximum isometric lifting weight and how long a subject could stand on one leg (One-legged Stand Test). All groups demonstrated a significant increase in back strength and the Nintendo Wii (VR) group was the only group that did not show a significant improvement in balance (18).

Specifically looking at sports performance in the setting of CLBP, Nambi et al. measured athlete agility before during and after intervention using the $40 \mathrm{~m}$ sprint, $4 \times 5$ sprint, submaximal shuttle run, vertical jump, countermovement jump, and squat jump. There was significant improvement for both the virtual reality group and the isokinetic training group for the $40 \mathrm{~m}$ sprint, $4 \times 5$ sprint, submaximal shuttle run, countermovement jump, and squat jump. A post hoc analysis showed more tendencies of improvement in the VRT group compared to Isokinetic Training (IKT) (17).

Karahan et al. were uniquely interested in Bath Ankylosing Spondylitis Functional Index (BAFSI) scores in their population. These improved significantly after 8 weeks of the VR program and remained unchanged in control group. Intergroup comparison after the 8th week showed significant improvement in exergame group BASFI scores (19).

\section{DISCUSSION}

Chronic low back pain as an entity is an immense societal cost and burden. Evidence-based treatment options are limited, despite decades of research on this subject. The standard of care 
TABLE 2 | Efficacy of VR to treat pain (top portion of Table) and functional change (bottom of portion of Table) associated with CLBP.

Theme: Efficacy of virtual reality to treat chronic low back pain

\begin{tabular}{|c|c|c|c|c|}
\hline Article title & First author & Study type & Measurement tool & Summary \\
\hline $\begin{array}{l}\text { An 8-Week Self-Administered At-Home } \\
\text { Behavioral Skills-Based Virtual Reality } \\
\text { Program for Chronic Low Back Pain: } \\
\text { Double-Blind, Randomized, } \\
\text { Placebo-Controlled Trial Conducted } \\
\text { During COVID-19 }\end{array}$ & Garcia & $\begin{array}{l}\text { Randomized } \\
\text { control trial } \\
(\mathrm{RCT})\end{array}$ & $\begin{array}{l}\text { DVPRS, DVPRSII, } \\
\text { PGIC, PCS, PSEQ2, } \\
\text { CPAQ-8 }\end{array}$ & $\begin{array}{l}\text { When compared to sham VR, the VR group } \\
\text { showed significantly lower average DVPRS scores } \\
\text { and significantly higher PGIC scores. No } \\
\text { significant differences between these groups were } \\
\text { observed for pain coping symptoms. }\end{array}$ \\
\hline $\begin{array}{l}\text { Virtual reality distraction induces } \\
\text { hypoalgesia in patients with chronic low } \\
\text { back pain: a randomized controlled trial }\end{array}$ & Matheve & RCT & NPRS, PCS & $\begin{array}{l}\text { There was significant reduction in pain intensity } \\
\text { during and after exercise for the VR group } \\
\text { compared to the control. }\end{array}$ \\
\hline $\begin{array}{l}\text { Comparative effects of isokinetic training } \\
\text { and virtual reality training on sports }\end{array}$ & Nambi & $\mathrm{RCT}$ & VAS & $\begin{array}{l}\text { The virtual reality group had a significant } \\
\text { improvement in VAS pain scores. }\end{array}$ \\
\hline
\end{tabular}

performances in university football

players with chronic low back

pain-randomized controlled study

Is physiotherapy integrated virtual

walking effective on pain, function, and

kinesiophobia in patients with

non-specific low-back pain?

Randomized controlled trial

After treatment the VR group showed a significant improvement in VAS pain scores.

improvement in VAS pain scores.

The effects of VR-based Wii Fit yoga on physical function in middle-aged female LBP patients

The effect of horse simulator riding on visual analogue scale, body composition and trunk strength in the patients with chronic low back pain

The effectiveness of exergames in patients with ankylosing spondylitis: a randomized controlled trial

The effects of the Nintendo Wii exercise program on chronic work-related low back pain in industrial workers

Virtual reality or isokinetic training; its effect on pain, kinesiophobia and serum stress hormones in chronic low back pain: A randomized controlled trial
Yilmaz

$$
\mathrm{RCT}
$$

VAS

Kim

Yoo

Karahan

RCT

Park

RCT

Nambi

RCT

VAS

Nambi

RCT

VAS

VAS

VAS

VAS

VAS
Short-Term Psychological and Hormonal Effects of Virtual Reality Training on

Chronic Low Back Pain in Soccer

Players
Theme: Efficacy of virtual reality to

treat functional change associated

with chronic low back pain

Feasibility and Safety of a Virtual Reality Dodgeball Intervention for Chronic Low Back Pain: A Randomized Clinical Trial

Efficacy of virtual reality to reduce

chronic low back pain: Proof-of-concept

of a non-pharmacological approach on

pain, quality of life, neuropsychological

and functional outcome
Thomas

$\mathrm{RCT}$

Alemanno

Case Control
Lumbar flexion excursion derived from Euler angles

RMDQ, truncal ROM derived from Euler angles, a Repetition Index derived for proprioception
No significant effects of group (game vs. control) on changes in lumbar spine flexion outside of gameplay.

Significant improvement in participation, trunk functionality, average range of motion, and proprioception at end of study compared to start. 
TABLE 2 | Continued

Theme: Efficacy of virtual reality to treat functional change associated

with chronic low back pain

\begin{tabular}{|c|c|c|c|c|}
\hline Article title & First author & Study type & Measurement tool & Summary \\
\hline $\begin{array}{l}\text { Is physiotherapy integrated virtual } \\
\text { walking effective on pain, function, and } \\
\text { kinesiophobia in patients with } \\
\text { non-specific low-back pain? } \\
\text { Randomised controlled trial }\end{array}$ & Yilmaz & $\mathrm{RCT}$ & $\begin{array}{l}\text { ODI, TUG, } 6 \mathrm{MWT}, \\
\text { single-leg balance } \\
\text { test }\end{array}$ & $\begin{array}{l}\text { After treatment, there was a significant difference } \\
\text { in the TUG and 6MWT scores between control } \\
\text { and experimental groups. No significant difference } \\
\text { in ODI or balance tests between groups, but there } \\
\text { was significant difference between pre and post } \\
\text { treatments in each group. }\end{array}$ \\
\hline $\begin{array}{l}\text { The effects of VR-based Wii Fit yoga on } \\
\text { physical function in middle-aged female } \\
\text { LBP patients }\end{array}$ & Kim & $\mathrm{RCT}$ & ODI and RMDQ & $\begin{array}{l}\text { Significant improvements between pre and post } \\
\text { training ODI and RMDQ scores. Significant } \\
\text { differences between ODI scores in groups and } \\
\text { significant differences in ODI and RMDQ with } \\
\text { regard to effect of time-by-group interaction. }\end{array}$ \\
\hline $\begin{array}{l}\text { The effect of horse simulator riding on } \\
\text { visual analogue scale, body composition } \\
\text { and trunk strength in the patients with } \\
\text { chronic low back pain }\end{array}$ & Yoo & $\mathrm{RCT}$ & $\begin{array}{l}\text { Total work and } \\
\text { isokinetic torque for } \\
\text { trunk flexion and } \\
\text { extension measured } \\
\text { with an isokinetic } \\
\text { dynamometer }\end{array}$ & $\begin{array}{l}\text { After } 8 \text { weeks, peak torque and total work were } \\
\text { significantly enhanced only in the horse simulator } \\
\text { riding group, and decreased in control group. } \\
\text { Related ratios correlated. }\end{array}$ \\
\hline $\begin{array}{l}\text { The effectiveness of exergames in } \\
\text { patients with ankylosing spondylitis: a } \\
\text { randomized controlled trial }\end{array}$ & Karahan & $\mathrm{RCT}$ & BASFI & $\begin{array}{l}\text { In exergame group, BASFI scores improved } \\
\text { significantly after } 8 \text { weeks of the program and } \\
\text { remained unchanged in control group. Intergroup } \\
\text { comparison after } 8 \text { th week showed significant } \\
\text { improvement in exergame group BASFI scores. }\end{array}$ \\
\hline $\begin{array}{l}\text { The effects of the Nintendo Wii exercise } \\
\text { program on chronic work-related low } \\
\text { back pain in industrial workers }\end{array}$ & Park & $\mathrm{RCT}$ & $\begin{array}{l}\text { Maximum isometric } \\
\text { lifting weight, One } \\
\text { legged stand test }\end{array}$ & $\begin{array}{l}\text { All groups demonstrated a significant increase in } \\
\text { back strength and the Nintendo Wii (VR) group } \\
\text { was the only group that did not show a significant } \\
\text { improvement in balance. }\end{array}$ \\
\hline $\begin{array}{l}\text { Comparative effects of isokinetic training } \\
\text { and virtual reality training on sports } \\
\text { performances in university football } \\
\text { players with chronic low back } \\
\text { pain-randomized controlled study }\end{array}$ & Nambi & $\mathrm{RCT}$ & $\begin{array}{l}40 \mathrm{~m} \text { sprint, } 4 \times 5 \\
\text { sprint, submaximal } \\
\text { shuttle run, Vertical } \\
\text { jump, } \\
\text { Countermovement } \\
\text { jump, Squat jump }\end{array}$ & $\begin{array}{l}\text { Significant improvement for virtual reality group } \\
\text { and IKT group for the } 40 \mathrm{~m} \text { sprint, } 4 \times 5 \text { sprint, } \\
\text { and submaximal shuttle run, CJ and SJ. A post } \\
\text { hoc analysis showed more tendencies of } \\
\text { improvement in the VRT group compared to IKT. }\end{array}$ \\
\hline
\end{tabular}

for the treatment of CLBP currently is multi-modal therapy, and yet this is still largely ineffective (4-6). Virtual reality is a novel treatment option with burgeoning evidence and may be an option for the physician's armamentarium.

In this scoping study, we used wide inclusion criteria and yet only 13 published studies were identified. This is a clear representation of the fact that research in this area is nascent. Future studies are required to further analyze the nature of the effect and safety of treatment of CLBP with VR.

The findings of this scoping study, while perhaps not fully generalizable, do offer several important conclusions. First, published data demonstrates safety and tolerance of VR for patients with CLBP, and that use of VR in treatment of CLBP seems to be feasible. Second, limited data suggests that VR therapies can improve quality of life in patients with CLBP and pain associated with CLBP. These findings did not, however, translate to efficacy of functional improvement as there is conflicting data as to whether functional measures improve with VR treatment. This data is very heterogeneous and therefore cannot be combined meaningfully to make succinct recommendations. This heterogeneity is found in both the variety of treatments utilized and the study populations in question.

In summary, this scoping review outlines existing literature regarding VR and CLBP demonstrates that four themes currently exist in this literature: safety and feasibility, impact on quality of life, impact on pain, and impact on function. It is obvious that much more research is necessary before recommendations can be made for or against the use of virtual reality therapies in the treatment of chronic low back pain. As VR research continues to develop, investigators should continue to include measures of CLBP pain and health-related quality of life consistent with those used in prior studies. These measures demonstrated sensitivity to change in the present scoping review and adding to extant data should strengthen outcomes. Measures of physical function are not as robust, so more work is needed to determine whether VR holds promise for physical rehabilitation in patients with CLBP. Although the nascent research uncovered in this scoping review found good evidence for safety and tolerability of VR, more studies of safety, acceptance, and satisfaction are recommended including focused studies of spinal pain risks specific to use of VR. Overall, the methodological quality of studies reviewed 
in this scoping review was poor and outcomes were limited to short-term posttreatment outcomes. Future studies are strongly encouraged to improve scientific rigor and include long-term follow-up assessments to more precisely gauge the durability of beneficial VR outcomes.

\section{REFERENCES}

1. Maher C, Underwood M, Buchbinder R. Non-specific low back pain. Lancet. (2017) 389:736-47. doi: 10.1016/S0140-6736(16)30970-9

2. Von Korff M. Studying the natural history of back pain. Spine. (1994) 19:20416S. doi: 10.1097/00007632-199409151-00005

3. Furlan AD, Pennick V, Bombardier C, van Tulder M, Editorial Board, Cochrane Back Review Group. 2009 updated method guidelines for systematic reviews in the Cochrane Back Review Group. Spine. (2009) 34:192941. doi: 10.1097/BRS.0b013e3181b1c99f

4. Urits I, Burshtein A, Sharma M, Testa L, Gold PA, Orhurhu V, et al. Low back pain, a comprehensive review: pathophysiology, diagnosis, and treatment. Curr Pain Headache Rep. (2019) 23:23. doi: 10.1007/s11916-019-0757-1

5. Mehra M, Hill K, Nicholl D, Schadrack J. The burden of chronic low back pain with and without a neuropathic component: a healthcare resource use and cost analysis. J Med Econ. (2012) 15:245-52. doi: 10.3111/13696998.2011.642090

6. Kreiner DS, Matz P, Bono CM, Cho CH, Easa JE, Ghiselli G, et al. Guideline summary review: an evidence-based clinical guideline for the diagnosis and treatment of low back pain. Spine J. (2020) 20:9981024. doi: $10.1016 /$ j.spinee.2020.04.006

7. Marr B. What is extended reality technology? A simple explanation for anyone. Forbes Magazine (2019).

8. Mallari B, Spaeth EK, Goh H, Boyd BS. Virtual reality as an analgesic for acute and chronic pain in adults: a systematic review and meta-analysis. J Pain Res. (2019) 12:2053-85. doi: 10.2147/JPR.S200498

9. Sumdani H, Aguilar-Salinas P, Avila MJ, Barber SR, Dumont TM. Utility of augmented reality and virtual reality in spine surgery: a systematic review of the literature. World Neurosurg. (2021). doi: 10.1016/j.wneu.2021.08.002

10. Hu H-Z, Feng XB, Shao ZW, Xie M, Xu S, Wu XH, et al. Application and prospect of mixed reality technology in medical field. Curr Med Sci. (2019) 39:1-6. doi: 10.1007/s11596-019-1992-8

11. Tack C. Virtual reality and chronic low back pain. Disabil Rehabil Assist Technol. (2021) 6:637-45. doi: 10.1080/17483107.2019.1688399

12. Gumaa M Youssef AR. Is virtual reality effective in orthopedic rehabilitation? A systematic review and meta-analysis. Phys Ther. (2019) 99:130425. doi: $10.1093 / \mathrm{ptj} / \mathrm{pzz} 093$

13. Arksey H O’Malley M. Scoping studies: towards a methodological framework. Int J Soc Res Method. (2005) 8:19-32. doi: 10.1080/1364557032000119616

14. Hennessy RW, Rumble D, Christian M, Brown DA, Trost Z. A graded exposure, locomotion-enabled virtual reality app during walking and reaching for individuals with chronic low back pain: cohort gaming design. JMIR Ser Games. (2020) 8:e17799. doi: 10.2196/17799

15. Thomas JS, France CR, Applegate ME, Leitkam ST, Walkowski S. Feasibility and safety of a virtual reality dodgeball intervention for chronic low back pain: a randomized clinical trial. J Pain. (2016) 17:130217. doi: 10.1016/j.jpain.2016.08.011

16. Nambi G, Abdelbasset WK, Alrawaili SM, Alsubaie SF, Abodonya AM, Saleh AK. Virtual reality or isokinetic training; its effect on pain, kinesiophobia and serum stress hormones in chronic low back pain: A randomized controlled trial. Technol Health Care. (2021) 29:155-166. doi: 10.3233/THC-202301

17. Nambi G, Abdelbasset WK, Elsayed SH, Alrawaili SM, Abodonya AM, Saleh $\mathrm{AK}$, et al. Comparative effects of isokinetic training and virtual reality training on sports performances in university football players with chronic low back pain-randomized controlled study. Evid Based Complement Altern Med. (2020) 2020:2981273. doi: 10.1155/2020/2981273

\section{AUTHOR CONTRIBUTIONS}

All authors contributed in depth to the literature review, analysis of the manuscripts, writing of the manuscript, and editing of the manuscript.

18. Park JH, Lee SH, Ko DS. The effects of the Nintendo Wii exercise program on chronic work-related low back pain in industrial workers. J Phys Ther Sci. (2013) 25:985-8. doi: 10.1589/jpts.25.985

19. Karahan AY, Tok F, Yildirim P, Ordahan B, Turkoglu G, Sahin N. The effectiveness of exergames in patients with ankylosing spondylitis: a randomized controlled trial. Adv Clin Exp Med. (2016) 25:9316. doi: $10.17219 /$ acem/32590

20. Alemanno F, Houdayer E, Emedoli D, Locatelli M, Mortini P, Mandelli C, et al. Efficacy of virtual reality to reduce chronic low back pain: proof-of-concept of a non-pharmacological approach on pain, quality of life, neuropsychological and functional outcome. PloS ONE. (2019) 14:e0216858. doi: 10.1371/journal.pone.0216858

21. Garcia LM, Birckhead BJ, Krishnamurthy P, Sackman J, Mackey IG, Louis RG, et al. An 8-week self-administered at-home behavioral skills-based virtual reality program for chronic low back pain: double-blind, randomized, placebo-controlled trial conducted during COVID-19. J Med Internet Res. (2021) 23:e26292. doi: 10.2196/26292

22. Matheve T, Bogaerts K, Timmermans A. Virtual reality distraction induces hypoalgesia in patients with chronic low back pain: a randomized controlled trial. J Neuroeng Rehabil. (2020) 17:55. doi: 10.1186/s12984-020-00688-0

23. Yilmaz Yelvar GD, Çirak Y, Dalkilinç M, Parlak Demir Y, Guner $\mathrm{Z}$, Boydak A. Is physiotherapy integrated virtual walking effective on pain, function, and kinesiophobia in patients with non-specific lowback pain? Randomised controlled trial. Eur Spine J. (2017) 26:53845. doi: 10.1007/s00586-016-4892-7

24. Kim S-S, Min W-K, Kim J-H, Lee B-H. The effects of VR-based Wii Fit yoga on physical function in middle-aged female LBP patients. J Phys Ther Sci. (2014) 26:549-52. doi: 10.1589/jpts.26.549

25. Yoo J-H, Kim S-E, Lee M-G, Jin JJ, Hong J, Choi YT, et al. The effect of horse simulator riding on visual analogue scale, body composition and trunk strength in the patients with chronic low back pain. Int J Clin Pract. (2014) 68:941-9. doi: 10.1111/ijcp.12414

26. Nambi G, Abdelbasset WK, Alsubaie SF, Saleh AK, Verma A, Abdelaziz MA, et al. Short-term psychological and hormonal effects of virtual reality training on chronic low back pain in soccer players. J Sport Rehabil. (2021) 1:1-10. doi: 10.1123/jsr.2020-0075

Conflict of Interest: The authors declare that the research was conducted in the absence of any commercial or financial relationships that could be construed as a potential conflict of interest.

Publisher's Note: All claims expressed in this article are solely those of the authors and do not necessarily represent those of their affiliated organizations, or those of the publisher, the editors and the reviewers. Any product that may be evaluated in this article, or claim that may be made by its manufacturer, is not guaranteed or endorsed by the publisher.

Copyright (c) 2022 Nagpal, Raghunandan, Tata, Kibler and McGeary. This is an open-access article distributed under the terms of the Creative Commons Attribution License (CC BY). The use, distribution or reproduction in other forums is permitted, provided the original author(s) and the copyright owner(s) are credited and that the original publication in this journal is cited, in accordance with accepted academic practice. No use, distribution or reproduction is permitted which does not comply with these terms. 